\title{
Comparing Impacts of Different Bowel Cleansing Agents and Oral Probiotics After Cleansing On Symptom and Microbiota: a Randomized Placebo-Controlled Trial
}

\section{Penghui Dai}

Department of Gastroenterology, The Affiliated Hospital of Southwest Medical University;

\section{Feng Tang}

Department of Gastroenterology, The Affiliated Hospital of Southwest Medical University;

Ke Gan

Department of Gastroenterology, The Affiliated Hospital of Southwest Medical University;

Qing Hu

Department of Gastroenterology, The Affiliated Hospital of Southwest Medical University;

\section{Jingyuan Liao}

Department of Gastroenterology, The Affiliated Hospital of Southwest Medical University;

\section{Xiaoyun Li}

Department of Gastroenterology, The Affiliated Hospital of Southwest Medical University;

Lei Shi

Department of Gastroenterology, The Affiliated Hospital of Southwest Medical University;

\section{Wei Zhang}

Department of Gastroenterology, The Affiliated Hospital of Southwest Medical University;

\section{Hailong Zhang}

Department of Gastroenterology, The Affiliated Hospital of Southwest Medical University;

\section{Qingliang Zhu}

Department of Gastroenterology, The Affiliated Hospital of Southwest Medical University;

Yongnan Cheng

Department of Gastroenterology, The Affiliated Hospital of Southwest Medical University;

Jie Zheng

Department of Gastroenterology, The Affiliated Hospital of Southwest Medical University;

Jie Chen

Department of Gastroenterology, The Affiliated Hospital of Southwest Medical University;

Ming Han

Department of Gastroenterology, The Affiliated Hospital of Southwest Medical University;

\section{Muhan Lv}

Department of Gastroenterology, The Affiliated Hospital of Southwest Medical University;

\section{Mingming Deng}

Department of Gastroenterology, The Affiliated Hospital of Southwest Medical University;

\section{Hongfen Xia}

The Affiliated Hospital of Southwest Medical University

Gang Luo ( $\square$ gangluo820@126.com )

Department of Gastroenterology, The Affiliated Hospital of Southwest Medical University;

\section{Research Article}

Keywords: mirabilite, probiotics, gut microbiota, bowel preparation, side effects

Posted Date: January 11th, 2022

DOl: https://doi.org/10.21203/rs.3.rs-1228536/v1 


\section{Abstract}

Background: Sodium sulfate-based purge has shown better quality of bowel preparation than polyethylene glycol (PEG) in a large retrospective study. However, its side effects and impact to gut microbiota have not been assessed. Besides, evidence from intestinal microecology that probiotics were beneficial to individuals who received bowel preparation is still lacking. The aims are to evaluate the side effects and microecological impact of two bowel cleansing agents (PEG and mirabilite), as well as the regulating effect of probiotics on microecosystem perturbed by bowel preparation.

Results: Mirabilite preparation appeared superior to PEG preparation in terms of side effects, consumed time and volume of solutions. Quantitative PCR results showed that the recovery rate of total microbial load in mirabilite group was faster than that in PEG group. 16S rRNA sequencing showed that there were no significant differences in effects of two bowel cleansing agents on multiple microbiota diversity metrics. And both laxatives may affect the relative abundance of core microbiota until 28 days after bowel preparation. Probiotics supplementation was beneficial to recovery of perturbed microecosystem and the maintenance of homeostasis in the gut according to our results. Moreover, probiotics supplementation relieved abdominal symptoms and few individual events induced by bowel preparation during long-term follow-up.

Conclusions: Mirabilite could be an optimal bowel cleansing agent for healthy people and can be applied broadly. Besides, probiotics are suggested to administrate after bowel cleansing as it brings multiple benefits in our study.

\section{Introduction}

Bowel preparation is an indispensable part of endoscopy procedure. Adequate bowel preparation significantly improves success rate of cecal intubation, adenoma positivity, and patient experience[1]. To this end, several laxatives have been extensively studied. The most common laxative used clinically is polyethylene glycol (PEG), which is considered to be an inert, safe and effective isotonic laxative. Furthermore, it has been recommended for use in multiple severe clinical conditions such as inflammatory bowel disease (IBD), renal failure, electrolyte imbalance, advanced liver disease, and poorly compensated congestive heart failure[1, 2]. Although PEG displayed excellent performance in terms of safety and efficacy, its impact to gut microbiota has remained elusive. With development of "Omics" technologies in recent years[3], the interplay of microbiota and pathogenesis of disease was able to thorough and deep study. By far, a number of diseases have been shown to have causal relationships with dysbiosis of intestinal microecology, ranging from enteric diseases such as IBD, irritable bowel syndrome (IBS), colorectal cancer, to cardiovascular, respiratory, hepatic and neurological diseases[4], etc. Besides, bowel preparation was also investigated due to a large volume of water rinsed out the majority of resident flora along with intestinal contents, potentially perturbing homeostasis of microecosystem, which in turn threatens human health. A previous study of bowel preparation based on healthy population showed that the abundance and composition of microbiota were sharply altered immediately after PEG purging, albeit transitory[5]. However, the implications of other bowel cleansing type on gut microbiota are not yet clear.

Maintaining homeostasis between host and intestinal microorganisms is of fundamental importance to human physiology and health[6]. Hence it is of great significance to seek an effective purgative agent with minor impact on the intestinal ecosystem. One of the most studied comparable laxatives is sodium phosphate (NaP), which has similar effect with PEG but better compliance. However, its side effects, principally dizziness and biochemical abnormalities, limited its application[2]. Mirabilite, a laxative widely used in Chinese medical institutions, is a potential option. The main component is sodium sulfafe decahydrate (Na2SO4 $\geq 99.3 \%$ ). It is stable appearing as a white, crystalline powder at room temperature and melts at 32.4 degrees Celsius[7]. The mechanism is to provide sulfate anions that are poorly absorbed in gastrointestinal tract, and the unabsorbed ions retain a large volume of ingested water, causing a copious watery diarrhea. The sodium sulfate-based preparation has been approved by FDA in 2010. And the efficacy was also evidenced by a large multicenter retrospective study with patients of 28,368 . Sodium sulfate-based preparation showed superior to PEG, sodium phosphate, and magnesium sulfate on quality of colonoscopy and detection rate of advanced adenoma, which had six times better quality of preparation than PEG based formulations[8]. Nonetheless, the side effects and impact of the sulfate-based agent to gut microbiota have not been investigated.

Probiotics functioning by maintaining intestinal microbial balance, have been suggested to be effective in multiple acute and chronic disorders[9]. Clinically, most common condition for probiotics supplementation is acute gastroenteritis, as evidence from several metaanalysis have shown that supplementation of probiotics containing L rhamnosus GG can shorten duration of diarrhea and hospitalization in children[10,11]. Other etiologies associated diarrhea including antibiotics[12], C. difficile infection[13], Irritable bowel syndrome (IBS)[14] have also been reported to benefit from probiotics either by reducing the risk or by alleviating the symptoms. A recent study based on 32 hospitalized patients has shown that probiotics can facilitate restoration of microbial diversity disrupted by PEG purging[15]. A systematic review of seven randomized controlled trials on healthy adults showed that there was a lack of evidence on the effects of probiotics on gut microbiome composition[16]. The effect of probiotics on microbial composition is controversial. 
The reasons can be ascribed to the diversity in inclusion criteria of subjects (healthy individuals or patients) and strains of probiotics supplementation. Currently, the outstanding issues are which laxative is ideal bowel cleansing agent for bowel preparation, and whether probiotics have a promising effect on restoring the microbiota after bowel preparation. For this purpose, we conducted a single-blind randomized control trial in a healthy population to attempt to shed light on these issues.

\section{Material And Methods}

\section{Study subject and design}

Subjects were recruited from the students at Southwest Medical University who met the following inclusion criteria, Han race, male or female, age between 18-26 years old, Body Mass Index (BMI) limited in 17-28 kg/ $\mathrm{m}^{2}$. The exclusion criteria included, administration of purgatives or prokinetic agents or antidiarrheal medications within a week, take probiotics or prebiotics within a month prior to experiment, use of antibiotics within 3 months prior to experiment, history of acute or chronic gastrointestinal diseases such as H. pylori infection, IBS, IBD, intestinal obstruction, intestinal bleeding, intestinal perforation, toxic megacolon, or appendix removed, etc., history of mental illnesses, poor compliance considered by researchers. Forty-three healthy volunteers who met the criteria were enrolled in the study. They are randomly assigned into two groups consuming PEG or mirabilite solution for bowel preparation. After bowel preparation, the subjects in both groups were randomized to take probiotics or placebo for 4 weeks, defined as M1 group (mirabilite and placebo), M2 group (mirabilite and probiotics), P1 group (PEG and placebo), and P2 (PEG and probiotics), respectively. Mirabilite was purchased from Sichuan Province Chuanmei Pharmaceutical Co., Ltd., Sichuan, China. The SFDA approval number is Z51022578. It contains nine packages (6g each), and the main ingredient is sodium sulfafe decahydrate as described. PEG was purchased from BEAUFOUR IPSEN Industrie Rue Ethe Virton-28100 Dreux, France. The SFDA approval number is $\mathrm{H} 20140560$. It comes in four packages, each containing polyethylene glycol $400064 \mathrm{~g}$, anhydrous sodium sulfate $5.7 \mathrm{~g}$, sodium bicarbonate $1.68 \mathrm{~g}$, sodium chloride $1.46 \mathrm{~g}$, and potassium chloride $0.75 \mathrm{~g}$. Probiotics used in this study are combined Bifidobacterium, Lactobacillus, Enterococcus and Bacillus cereus Tablets (SiLianKang), each tablet containing Bifidobacterium infantis $>0.5 \times 10^{6}$ CFU, Lactobacillus acidophilus $>0.5 \times 10^{6} \mathrm{CFU}$, Enterococcus faecalis $>0.5 \times 10^{6} \mathrm{CFU}$, Bacillus cereus $>0.5 \times 10^{5} \mathrm{CFU}$, the probiotics and placebo were gifted by Hangzhou Grand Biologic Pharmaceutical INC., Hangzhou, China. The SFDA approval number is S20060010.

Subjects were gathered to perform the bowel preparation in batches. All subjects signed an informed consent form prior to bowel preparation with either mirabilite or PEG as designed. For use of mirabilite, nine packages $(54 \mathrm{~g})$ dissolved into $200 \mathrm{~mL}$ warm water (temperature above 32.4 degrees Celsius). Participants drank the solution in 30 minutes and 2 liters of water as designed (drinking water until last effluent with clear liquid) in the following 2 hours. PEG is used according to the instructions, that is, one package dissolved into 1 liter of water, and participants drank 1 liter of PEG electrolyte solution for every $20 \mathrm{~kg}$ of body weight as designed (drinking enough electrolyte solution until last effluent with clear liquid). The actual drinking volume of water or solution, frequency of defecation, consumption time, and adverse events of bowel preparation were recorded by two experienced physicians.

Probiotics or placebo were consumed three times a day, 3 tablets each time administrated after meals. Stool sample collections were designed in four points, 1 day before bowel cleansing, 1, 14, and 28 days after bowel cleansing. Participants received probiotics or placebo after completed the second time of sample collection. Participants were suggested to have a regular routine and avoid alcohol or spicy food. Individuals consuming antibiotics or other probiotics supplements during the follow-up period will be drop out the study. Sample collected at student dormitory with two germfree tubes. Collected samples were delivered to the fecal microbiota transplantation center for storage at $-80^{\circ} \mathrm{C}$ within 15 minutes.

\section{Quantitative PCR}

Quantitative PCR was used to measure the absolute number of bacteria in total of 160 fecal samples through targeting 16S rRNA gene. A human gene (beta-actin) was employed as internal control. The protocol for quantitative PCR was described previously[17]. Briefly, the samples were weighted and extracted the genomic DNA. Then 16S gene was amplified and constructed into pMD18-T vector. Quantitative PCR was performed on diluted plasmid to construct standard curve. The $16 \mathrm{~S}$ gene was analyzed by quantitative PCR and the copy number was determined by standard curve. The detailed reagents and reaction condition were shown in Table S1.

\section{Sequencing and data analysis}

Total genome DNA from samples was extracted using CTAB method. DNA concentration and purity were monitored on $1 \%$ agarose gels. Then, DNA was diluted to $1 \mathrm{ng} / \mu \mathrm{L}$ using sterile water according to the concentration. $16 \mathrm{~S}$ rRNA genes of V4 region was amplified used primer $515 \mathrm{~F}$ 806R with the barcode. The PCR products were extracted from 2\% agarose gel and purified with Qiagen Gel Extraction Kit (Qiagen, Germany). Sequencing libraries were generated using TruSeq ${ }^{\circledR}$ DNA PCR-Free Sample Preparation Kit (Illumina, USA) following manufacturer's 
instructions and index codes were added. The library quality was assessed on the Qubit@ 2.0 Fluorometer (Thermo Scientific) and Agilent Bioanalyzer 2100 system. At last, the library was sequenced on an Illumina NovaSeq platform and 250 bp paired-end reads were generated.

Sequences analysis were performed by Uparse software (Uparse v7.0.1001, http://drive5.com/uparse/)[18]. Sequences with $\geq 97 \%$ similarity were assigned to the same OTUs. Representative sequence for each OTU was screened for further annotation. The Silva Database (http://www.arb-silva.de/)[19] was used based on Mothur algorithm to annotate taxonomic information. In order to study phylogenetic relationship of different OTUs, and the difference of the dominant species in different samples (groups), multiple sequence alignment was conducted using the MUSCLE software (Version 3.8.31, http://www.drive5.com/muscle/)[20]. OTUs abundance information were normalized using a standard of sequence number corresponding to the sample with the least sequences. Subsequent analysis of alpha diversity and beta diversity were all performed basing on this output normalized data.

Alpha diversity is applied in analyzing complexity of bacterial diversity within a sample, of which 5 indices were employed in this study, including Observed-species, Chao1, Simpson, Shannon, Good-coverage. All these indices were calculated with QIIME (Version 1.7.0), and a Wilcoxon rank sum test was used to determine the differences in species diversity between groups within samples. Beta diversity analysis was used to assess similarities in bacterial composition between samples. Principal Coordinate Analysis (PCoA) was performed to get principal coordinates and visualize from complex, multidimensional data. A distance matrix of weighted or unweighted unifrac among samples obtained before was transformed to a new set of orthogonal axes, by which the maximum variation factor is demonstrated by first principal coordinate, and the second maximum one by the second principal coordinate. PCoA analysis was displayed by WGCNA package, stat packages and ggplot2 package in R software (Version 2.15.3). Beta diversity on both weighted and unweighted unifrac distance were analyzed on QIIME software (Version 1.9.1), and differences in bacterial composition between groups were determined by adonis test.

LEfSe (Linear discriminant analysis Effect Size) was used to determine the most abundant species between groups. Parametric kruskal-wallis (KW) sum-rank test (non-parametric kruskal-wallis and rank test) was used to determine the species with significant differences in relative abundance between groups. Then, a Wilcoxon rank sum test was used to determine the difference between groups. Finally, linear discriminant analysis (LDA) was used to reduce and evaluate the impact of significantly different species (LDA Score).

\section{Statistical analysis}

Data analysis was performed in GraphPad Prism software version 9.0 (GraphPad Inc, CA, USA) and SPSS software version 25.0 (IBM, NY, USA). Continuous variables are expressed as mean \pm standard deviation, and categorical variables are expressed as number and percentage. Continuous variables were analyzed by independent samples t-test (two tailed) or covariance analysis (baseline as covariate). For analysis of categorical variables, Chi-square and Fisher's exact test were used to compare difference between 2 groups. A p value $<0.05$ was statistically significant.

\section{Results}

\section{Study subjects}

The study design is shown in Figure 1. A total of forty-three healthy volunteers completed the bowel cleansing either with mirabilite or PEG according to experimental design. Three subjects failed to continue the study during follow-up, two of whom came down with colds and requested to discontinue the study; another one was dropped out due to administration of antibiotics when suffering a urinary tract infection. The remaining forty subjects completed throughout the study. Demographic characteristics of subjects, including age, gender, height, body weight, and BMI, were homogeneous distributed in all experimental groups (Table 1).

\section{Adverse events related to bowel cleansing}

The laxatives bring about an unpleasant set of symptoms to patients, we first compared the side effects of those two laxatives. As shown in Table 2, PEG group showed significant more frequent nausea and vomiting than mirabilite $(36.84 \%$ vs $0 \%, p=0.024,76.19 \%$ vs $33.33 \%$, $p=0.009$, respectively). The frequency of other adverse events, including bloating, abdominal pain, dizziness, headache, chest distress, were not significantly differences between the two groups. Moreover, as shown in Table S2, the average amount of PEG solution consumed was $2724 \mathrm{ml}$, while consumed mirabilite solution was $1876 \mathrm{ml}$, PEG group consumed nearly 1.5 times as much solution as the mirabilite group $(p<0.001)$. Meanwhile, PEG group consumed longer time to complete comparing with mirabilite group, though not statistically significant (PEG vs mirabilite, $1.73 \mathrm{hr}$ vs $1.42 \mathrm{hr}, p=0.083)$. Nevertheless, there was not difference in frequency of defecation between the two groups when the effluent achieved clear liquid (5 vs 5 times, $p=0.378$ ).

\section{The effect of laxatives on total microbial load}


Previous study suggests that total microbial load decreased 34.7-fold immediately after bowel cleansing by PEG purging and recovered in 14 days[5]. To compare the effect of those two laxatives on total microbial load, quantitative PCR was used to measure the copy number of gut microorganisms. As shown in Figure 2A-D, the copy number of gut microorganisms was dramatically dropped on both PEG and mirabilite group in first day after bowel cleansing when compared with baseline, but the dropped number was quickly restored to baseline within 14 days. Next, when we compared the first day follow up samples between PEG with mirabilite group, found that the number of gut microorganisms in PEG group was significantly lower than that in mirabilite group (Figure 2E). To verify this result, a human gene (beta-actin), as an internal control, was examined in the fecal samples. As shown in Figure 2F, there was no significant difference in the copy number of beta-actin between each group. These results suggested that the recovery rate after purging with mirabilite may be faster than that of PEG purging.

\section{Changes in the microbiota composition}

A total of 16,248,023 raw sequences were generated in one hundred and sixty samples. Moving out low quality, short length (166,813) and chimeric $(78,686)$ sequences obtained $16,002,524$ effective sequences with an average number of 100,015 per sample at an average length of 253 bps per sequence. To study the species composition of samples, the effective sequences were clustered into 11706 0TUs based on their shared sequence identity at a $97 \%$ threshold. Sequencing depth was assessed by Rarefaction Curve and Species accumulation boxplot (Figure S1A-B), both of which indicating that the sequencing data was sufficient to analyze the species diversity.

Alpha diversity measures the taxa richness and evenness within individual. To determine whether purgatives lavage and probiotics supplementation affected taxa diversity, we first assessed taxa richness through OUT-base (Chao1) and phylogenetic tree-based methods (PDWT). As shown in Figure 3A-D \& Figure S2A-D, richness was no significant difference between pre- and post- bowel cleansing samples in both

P1 and M1 group. Probiotics supplementation for 28 days increased taxa richness in PEG purging group (Figure 3B \& Figure S2B), but reduced in mirabilite purging group comparing with baseline (Figure 3D \& Figure S2D) (Chao1 and PD-WT, all comparisons, $p<0.05$ ). Shannon index, a quantitative measure of biodiversity reflecting taxa richness and evenness, was used to assess alpha diversity in this study. As shown in Figure 3E-F \& Figure S2A-D, there were no significant differences in Shannon index as well as Simpson index in all experimental group (all comparisons, $p>0.05)$.

Beta diversity is a measure of similarity or dissimilarity in diversity between two communities. We used Principal coordinate analysis (PCoA) to visualized microbiota community structure based on weighted and unweighted unifrac distance matrices. On weighted unifrac distance, a similar clustering of samples from baseline and different follow up time points was observed in all experimental group, except for 28 days follow up samples in P2 group deviated from baseline (pseudo $F=3.457, R^{2}=0.1611, p=0.017$ ) (Figure 4A-D). Unlike weighted unifrac distance matrices, which considered both OTU abundances and presence/absence, the unweighted unifrac distance matrices only take into account OTU presence/absence. As shown in Figure S3A, a significant different cluster was observed from all follow up samples and baseline in P1 group (all comparisons, $p<0.05$ ), whereas a similar cluster was observed in M1 group (Figure S3C) (all comparisons, $p>0.05$ ). Probiotics supplementation resulted in a remarkable separation of all follow up samples from baseline in both P2 and M2 group (Figure S3B \& Figure S3D) (all comparisons, $p<0.05$ ), except for 14 days follow up samples in $\mathrm{P} 2$ group (pseudo $F=1.574, R^{2}=0.0804, p=0.124$ ). These results indicated that PEG purging may affect growth of rare taxa, while mirabilite probably have less effect, and probiotics may have great impact on rara taxa.

In addition, we used PCoA to analyze the taxa similarities of demographic characteristics including age, sex and BMI. There were no significant differences in age and sex on weighted and unweighted unifrac distance matrices (Figure S4A-B \& Figure S4D-E). Previous studies suggested that obese and lean subjects presented different microbiota composition profile, and diversity was reduced in obese subjects[21, 22]. Our cohort contained 3 overweight subjects, 25 normal weight subjects, and 12 underweight subjects. On weighted unifrac distance, underweight and normal weight cohort presented similar microbial community structure, whereas overweight cohort showed significant difference microbial community structure with normal weight and underweight cohort (Figure S4C \& S4F).

\section{Specific microbiota change}

The top five dominant phyla in the baseline samples were Bacteroidetes, Firmicutes, Proteobacteria, Actinobacteria and Fusobacteriota, with relative abundance of $48 \%, 40 \%, 5 \%, 3 \%$ and $0.4 \%$, respectively. The microbiota composition was consistent with previous report on Chinese healthy population. LEfSe analysis was employed to determine the difference in microbiota. At phylum level, Fusobacteriota was observed more abundant in first day follow-up samples in PEG group, and Acidobacteriota and Verrucomicrobiota were less abundant in first day followup samples in mirabilite group. At genus and species level, the relative abundance of Holdemanella, Lactobacillus_reuteri, Blautia,

Ruminococcus_torques_group, Bacteroides_caecigallinarum, and Fusobacterium_mortiferum were enriched in the first day follow-up samples in PEG group compared with baseline, and the reduced taxa in relative abundance were UCG_002 and Bacteroides_uniformis (Figure 5A). In mirabilite group, Anaerostipes_hadrus, Butyricicoccus, Lachnoclostridium, Romboutsia ilealis, Ruminococcus_torques_group, and 
Parabacteroides were significantly enriched in the first day follow up samples when compared with baseline (Figure 5C). The cladogram revealed the differences in the phylogenetic distribution of taxa between pre- and post- bowel preparation (Figure 5B\&D).

We then investigated whether probiotics supplementation was beneficial to the recovery of altered microbiota after bowel cleansing. As shown in Table 3, in placebo group, most taxa restored to baseline within 14days after PEG purging, except for Lactobacillus_reuteri, UCG_002 and Bacteroides_caecigallinarum. In probiotics group, the taxa basically recovered within 14days after PEG purging, except for Holdemanella and UCG_002. By 28 days of follow-up, only UCG_002 in P1 group and Ruminococcus_torques_group in P2 group were significantly reduced in relative abundance compared with baseline. As shown in Table 4, after lavage with mirabilite, only the probiotics group, but not the placebo group, recovered essentially within 14days. By 28 days of follow-up, both groups achieved utterly recovery. These results suggest that probiotics supplementation was instrumental for recovery of microbiota after bowel preparation.

\section{Short-and long-term follow-up}

To determine whether bowel preparation affected short- and long-term health, a questionnaire about abdominal symptoms and other selfreported individual events was designed. All subjects filled out the questionnaires at one month and one year. As shown in Table S3, the total abdominal symptoms scores were observed to elevate in M1 and P1 group after one month of bowel preparation, and probiotics tended to improve the scores in both mirabilite and PEG group, though not statistical difference $(p=0.053, p=0.340$, respectively). A comparison of abdominal symptoms scores between mirabilite and PEG group revealed no significant difference at one month and one year $(p=0.243$, $\mathrm{p}=0.750$, respectively). The total abdominal symptoms scores returned to baseline after one year at both mirabilite and PEG group. BMI showed no significant difference between each group after a year compared with baseline.

Several individual events, included spicy intolerance, increased appetite, and increased defecation frequency, were inquired based on selfreports (Table S4). Few subjects reported intolerance to spicy after bowel preparation in M1 and P1 group, probiotics administration seemed to reduce the reporting rate in both mirabilite and PEG group. Quite a few subjects reported to increase appetite after bowel preparation in mirabilite and PEG group, especially in M2 group. And many subjects reported increased frequency of defecation (1 time per day), this phenomenon was more common in P2 group and was remained at a year later for few subjects.

\section{Discussion}

PEG is officially recommended bowel cleansing agent and does not cause water and electrolyte imbalance because it is an isotonic solution. However, plastic-like taste and large volume of solution induced a high frequency of adverse events (mainly nausea and vomiting), resulting in patient intolerance and failure of bowel cleansing. Many studies have attempted to improve the taste through adding orange juice[23], pineapple juice[24], and coke zero[25] to various regimes of PEG. Although these additives increase palatability and decrease adverse events, they still have limitations, such as diabetics. Mirabilite solution is hyperosmotic, the osmotic pressure is nearly ten times that of plasma, which stimulates intense intestinal peristalsis. Following drink 2 liters of water to maintain osmotic balance and rinse intestinal tract. As such, mirabilite does not induce water and electrolyte imbalance. Besides, mirabilite with the salty tastes is well tolerated for most of subjects, the frequency of nausea and vomiting in our study are 36.84\% and 0, respectively, far lower than that of PEG with $76.19 \%$ and $33.33 \%$, respectively. Moreover, the salty tastes and hyperosmotic solution inspired thirst for water, reduced the time of bowel preparation. Therefore, mirabilite is a safe, effective, and well tolerated purgative agent for bowel preparation.

Bowel preparation flushed out most gut bacteria, the microbial load reduced 25.3-fold immediately after purgative treatment with PEG, but it quickly restored to baseline within 14 days[5]. This result is accordant with our study observed on PEG. Surprisingly, mirabilite group showed faster recovery rate than that of PEG group, with significantly more bacterial load in the first day follow-up samples. At this moment, microbial load reached a third of baseline level in mirabilite group, while the PEG group restored only one-sixth. The reason may be attributed to the amount of solution consumed. PEG group consumed 1 liter more solution on average than the mirabilite group, resulting in greater scouring effect on intestinal tract. Despite the number of total bacteria was varied, the microbiota composition of both groups was resembled to baseline on the first day after bowel preparation. Previous study reported that approximately $22 \%$ healthy subjects altered microbiota composition immediately after bowel preparation, and all these subjects restored to baseline within 14 days[5]. Similarly, Naoyoshi et al. reported on healthy subjects that microbiota composition is reduced immediately after bowel preparation, but not at 14 days follow up samples[26]. Rima M et al. revealed that bowel preparations affected the composition and diversity of the fecal and luminal microbiota in the short term (less than 7days)[27]. Our study suggested that microbiota composition can be restored as early as the first day after bowel preparation for healthy people.

Although the microbiota composition is quickly restored, a few taxa were observed to be affected in the relative abundance on the first day after bowel preparation. These taxa increased in PEG group included Holdemanella and Blautia that producing short-chain fatty acids, Lactobacillus_reuteri[28] (probiotics) producing antimicrobial molecules, and Fusobacterium_mortiferum[29] (opportunistic pathogen), etc. 
Similarly, taxa increased in mirabilite group contained beneficent bacteria such as Anaerostipes_hadrus[30], Butyricicoccus[31], and harmful bacteria such as Parabacteroides[32], Ruminococcus_torques_group[33]. The microbiota composition is highly subject-specific depending on age, diet, geography, race and ethnicity, etc. The core microbiome that shared species between individuals only account for 30\%[34]. This may explain the inconsistencies in change of microbiota by laxatives treatment across studies[5, 26]. In our study, ninety (30.7\%) OTU were shared in all 40 individuals, some of which were observed in above taxa with relative abundance change on both PEG and mirabilite group. This result indicated that bowel preparation affected core microbiome in gut, albeit temporarily. In our study, the relative abundance of microbiota can be fully restored to baseline within 28 days on both PEG and mirabilite group.

A little is known about long term impacts of bowel preparation. In this study, abdominal symptoms and several individual events were documented at one month and one year after bowel preparation. The results showed that bowel preparation increased abdominal symptoms scores, reports rate of intolerance to spicy foods, appetite, stool frequency, and moisture of stool. We speculate that these symptoms are related to microbial changes and incomplete recovery of intestinal function. Bowel preparation flushed out the majority of luminal bacteria and reduced the number of bacteria in intestinal mucosa (number of OTUs and richness was decreased in post-prep biopsy samples[27]).

Together with disturbed microecosystem, the intestinal mucosa barrier, which is a thick layer of mucus harbored large amounts of commensal bacteria that prevents the pathogen and other toxic substances from reaching the epithelial surface[35], was impaired during bowel preparation, and thus weak immune system that may attribute to some of above clinic manifestations. Probiotics have been shown to be beneficial for a variety of human conditions[36]. In our study, probiotics supplementation relieved abdominal symptoms and some individual events induced by bowel preparation. Several actions have been suggested to be mechanism of probiotics, such as producing antimicrobial substances, stimulating mucosa secretion, and strengthening integrity of gut barrier to maintain homeostasis in gut mucosa[35, 37]. Evidence from our study supported that probiotics supplementation promoted the recovery of microbial abundance and maintained the homeostasis of hosts. Meanwhile, probiotics in our study failed to affect the gut microbiota composition in health adults, which is consistent with a previous systematic review[16].

\section{Conclusions}

In summary, a previous retrospective study has suggested that sodium sulfate-based preparation had better quality of preparation than PEG, but its side effects and microbial alteration have not been reported. We reported for the first time that mirabilite preparation appeared superior to PEG preparation in terms of side effects and total microbial load recovery. In addition, there were no significant differences in effects of two laxatives on multiple microbiota diversity metrics. And both laxatives affected the relative abundance of core microbiota until 28 days after bowel preparation. Probiotics supplementation was beneficial to recovery of disturbed microecosystem and maintained homeostasis in the gut according to our results. Moreover, probiotics supplementation relieved abdominal symptoms and few individual events induced by bowel preparation during long-term follow-up. Our study concludes that mirabilite could be an optimal bowel cleansing agent for healthy people who is about to undergo a colonoscopy, and probiotics are suggested after bowel preparation as it brings multiple benefits.

\section{Abbreviations}

PEG:Polyethylene glycol; PCR:Polymerase chain reaction; IBD:Inflammatory bowel disease; IBS:Irritable bowel syndrome; NaP:Sodium phosphate; Na2SO4:Sodium sulfafe decahydrate; BMI:Body Mass Index; FDA:Food and Drug Administration; SFDA:State Food and Drug Administration; rRNA:Ribosomal RNA; OUT:Operational taxonomic unit; PCoA:Principal coordinate analysis; LEfSe:Linear discriminant analysis effect size; KW:Kruskal-wallis; LDA:Linear discriminant analysis; PD-WT:Phylogenetic diversity-whole tree

\section{Declarations}

\section{Acknowledgements}

We thank all volunteers for participation and cooperation. We thank Dr. Run Chen (Department of Statistics, Southwest Medical University) for her assistance in data analysis. We thank Sichuan Province Chuanmei Pharmaceutical Co., Ltd for the gift of mirabilite. We thank Hangzhou Grand Biologic Pharmaceutical INC for gift of probiotics and placebo. We thank Majorbio Bio-pharm Technology Co., Ltd (Shanghai, China)for technical support of quantitative PCR.

\section{Funding}

This work was supported by the grants from Luzhou People's Government-Southwest Medical University Science and Technology Strategic Cooperation Project (2018LZXNYD-ZK24) and the foundation of Luzhou Science and Technology Bureau (2019-JYJ-61).

\section{Availability of data and materials}


The datasets of the 16S rRNA in our study are available in the Sequence Read Archive (SRA) under project number PRJNA793639,but these data are still review.

\section{Author contributions}

GL, MMD, and HFX conceived and designed the study. PHD, FT, and KG recruited the healthy volunteers, collected the samples, and followed up. PHD, FT, KG and GL analyzed the data. YNC, JZ, JC, and MH performed bioinformatic analysis. QH, JYL, XYL, LS, WZ, HLZ, QLZ, and MHL provided intellectual contribution. GL wrote the manuscript. All authors read and participated in revision of the manuscript.

\section{Author details}

${ }^{1}$ Department of Gastroenterology, The Affiliated Hospital of Southwest Medical University, Luzhou 646000 , Sichuan, China; ${ }^{2}$ Department of Endoscopic Medicine, The Affiliated Hospital of Southwest Medical University, Luzhou 646000 , Sichuan, China

\section{Ethics approval and Consent to participate}

The study protocol was approval by the ethics committee of the affiliated hospital of Southwest Medical University. Written informed consent was obtained from healthy volunteers prior to bowel preparation.

\section{Consent for publication}

Not applicable.

\section{Competing interests}

The authors declare no competing interests.

\section{References}

1. Hassan, C., et al., Bowel preparation for colonoscopy: European Society of Gastrointestinal Endoscopy (ESGE) Guideline - Update 2019. Endoscopy, 2019. 51(8): p. 775-794.

2. Parra-Blanco, A., et al., Achieving the best bowel preparation for colonoscopy. World J Gastroenterol, 2014. 20(47): p. 17709-26.

3. Segal, J.P., et al., The application of omics techniques to understand the role of the gut microbiota in inflammatory bowel disease. Therap Adv Gastroenterol, 2019. 12: p. 1756284818822250.

4. Carding, S., et al., Dysbiosis of the gut microbiota in disease. Microb Ecol Health Dis, 2015. 26: p. 26191.

5. Jalanka, J., et al., Effects of bowel cleansing on the intestinal microbiota. Gut, 2015. 64(10): p. $1562-8$.

6. Hooper, L.V. and A.J. Macpherson, Immune adaptations that maintain homeostasis with the intestinal microbiota. Nat Rev Immunol, 2010. 10(3): p. 159-69.

7. Benington, F., Preliminary Identification of Crystalline Phases in a Transparent Stalactite. Science, 1959. 129(3357): p. 1227.

8. Martin, D., et al., Impact of bowel preparation type on the quality of colonoscopy: a multicenter community-based study. J Community Hosp Intern Med Perspect, 2016. 6(2): p. 31074.

9. Sanders, M.E., et al., Probiotics and prebiotics in intestinal health and disease: from biology to the clinic. Nat Rev Gastroenterol Hepatol, 2019. 16(10): p. 605-616.

10. Szajewska, H., et al., Systematic review with meta-analysis: Lactobacillus rhamnosus GG for treating acute gastroenteritis in children - a 2019 update. Aliment Pharmacol Ther, 2019. 49(11): p. 1376-1384.

11. Li, Y.T., et al., Efficacy of Lactobacillus rhamnosus GG in treatment of acute pediatric diarrhea: A systematic review with meta-analysis. World J Gastroenterol, 2019. 25(33): p. 4999-5016.

12. Cremonini, F. and E.J. Videlock, Probiotics are associated with a decreased risk of antibiotic-associated diarrhoea. Evid Based Med, 2013. 18(2): p. 71-2. 
13. Allen, S.J., et al., Lactobacilli and bifidobacteria in the prevention of antibiotic-associated diarrhoea and Clostridium difficile diarrhoea in older inpatients (PLACIDE): a randomised, double-blind, placebo-controlled, multicentre trial. Lancet, 2013. 382(9900): p. $1249-57$.

14. Ford, A.C., et al., Systematic review with meta-analysis: the efficacy of prebiotics, probiotics, synbiotics and antibiotics in irritable bowel syndrome. Aliment Pharmacol Ther, 2018. 48(10): p. 1044-1060.

15. Deng, X., et al., Oral Probiotics Alleviate Intestinal Dysbacteriosis for People Receiving Bowel Preparation. Front Med (Lausanne), 2020. 7: p. 73.

16. Kristensen, N.B., et al., Alterations in fecal microbiota composition by probiotic supplementation in healthy adults: a systematic review of randomized controlled trials. Genome Med, 2016. 8(1): p. 52.

17. Zemb, O., et al., Absolute quantitation of microbes using 16S rRNA gene metabarcoding: A rapid normalization of relative abundances by quantitative PCR targeting a 16S rRNA gene spike-in standard. Microbiologyopen, 2020. 9(3): p. e977.

18. Edgar, R.C., UPARSE: highly accurate OTU sequences from microbial amplicon reads. Nat Methods, 2013. 10(10): p. 996-8.

19. Quast, C., et al., The SILVA ribosomal RNA gene database project: improved data processing and web-based tools. Nucleic Acids Res, 2013. 41(Database issue): p. D590-6.

20. Edgar, R.C., MUSCLE: multiple sequence alignment with high accuracy and high throughput. Nucleic Acids Res, 2004. 32(5): p. $1792-7$.

21. Muscogiuri, G., et al., Gut microbiota: a new path to treat obesity. Int J Obes Suppl, 2019. 9(1): p. 10-19.

22. Boroni Moreira, A.P., et al., Gut microbiota and the development of obesity. Nutr Hosp, 2012. 27(5): p. $1408-14$.

23. Choi, H.S., et al., Orange juice intake reduces patient discomfort and is effective for bowel cleansing with polyethylene glycol during bowel preparation. Dis Colon Rectum, 2014. 57(10): p. 1220-7.

24. Altınbas, A., et al., Adding pineapple juice to a polyethylene glycol-based bowel cleansing regime improved the quality of colon cleaning. Ann Nutr Metab, 2013. 63(1-2): p. 83-7.

25. Seow-En, I. and F. Seow-Choen, A prospective randomized trial on the use of Coca-Cola Zero(®) vs water for polyethylene glycol bowel preparation before colonoscopy. Colorectal Dis, 2016. 18(7): p. 717-23.

26. Nagata, N., et al., Effects of bowel preparation on the human gut microbiome and metabolome. Sci Rep, 2019. 9(1): p. 4042.

27. Shobar, R.M., et al., The Effects of Bowel Preparation on Microbiota-Related Metrics Differ in Health and in Inflammatory Bowel Disease and for the Mucosal and Luminal Microbiota Compartments. Clin Transl Gastroenterol, 2016. 7: p. e143.

28. Mu, Q., V.J. Tavella, and X.M. Luo, Role of Lactobacillus reuteri in Human Health and Diseases. Front Microbiol, 2018.9 : p. 757.

29. He, W.Q., et al., Composition of gut and oropharynx bacterial communities in Rattus norvegicus and Suncus murinus in China. BMC Vet Res, 2020. 16(1): p. 413.

30. Kant, R., et al., Genome Sequence of the Butyrate-Producing Anaerobic Bacterium Anaerostipes hadrus PEL 85. Genome Announc, 2015. $3(2)$.

31. Devriese, S., et al., Reduced Mucosa-associated Butyricicoccus Activity in Patients with Ulcerative Colitis Correlates with Aberrant Claudin-1 Expression. J Crohns Colitis, 2017. 11(2): p. 229-236.

32. Meng, X., et al., Gut dysbacteriosis and intestinal disease: mechanism and treatment. J Appl Microbiol, 2020. 129(4): p. 787-805.

33. Deaver, J.A., S.Y. Eum, and M. Toborek, Circadian Disruption Changes Gut Microbiome Taxa and Functional Gene Composition. Front Microbiol, 2018. 9: p. 737.

34. Faith, J.J., et al., The long-term stability of the human gut microbiota. Science, 2013. 341(6141): p. 1237439.

35. Cornick, S., A. Tawiah, and K. Chadee, Roles and regulation of the mucus barrier in the gut. Tissue Barriers, 2015. 3(1-2): p. e982426.

36. Han, S., et al., Probiotic Gastrointestinal Transit and Colonization After Oral Administration: A Long Journey. Front Cell Infect Microbiol, 2021. 11: p. 609722.

Page $10 / 16$ 
37. Plaza-Diaz, J., et al., Mechanisms of Action of Probiotics. Adv Nutr, 2019. 10(suppl_1): p. S49-S66.

\section{Tables}

Table 1 Demographic characteristics of study subjects

\begin{tabular}{|c|c|c|c|c|c|}
\hline \multirow[b]{2}{*}{ Variable } & \multicolumn{2}{|l|}{ Mirabilite } & \multicolumn{2}{|l|}{ PEG } & \multirow[b]{2}{*}{$P$-value } \\
\hline & Probiotics (M1 group) & $\begin{array}{l}\text { Placebo } \\
\text { (M2 group) }\end{array}$ & $\begin{array}{l}\text { Probiotics } \\
\text { (P1 group) }\end{array}$ & $\begin{array}{l}\text { Placebo } \\
\text { (P2 group) }\end{array}$ & \\
\hline Age (years) & $22.4 \pm 1.50$ & $21.4 \pm 1.17$ & $21.9 \pm 1.73$ & $21.9 \pm 1.34$ & 0.460 \\
\hline Gender (male/female) & $2 / 7$ & $3 / 7$ & $2 / 8$ & $3 / 8$ & 0.991 \\
\hline Height (m) & $1.62 \pm 0.08$ & $1.67 \pm 0.06$ & $1.62 \pm 0.09$ & $1.63 \pm 0.09$ & 0.415 \\
\hline Body weight (kg) & $53.6 \pm 10.99$ & $56.6 \pm 6.15$ & $53.2 \pm 12.06$ & $53.7 \pm 8.64$ & 0.856 \\
\hline BMI $\left(\mathrm{kg} / \mathrm{m}^{2}\right)$ & $20.47 \pm 2.60$ & $20.37 \pm 1.71$ & $20.22 \pm 2.01$ & $20.01 \pm 2.89$ & 0.974 \\
\hline
\end{tabular}

BMI, body mass index; PEG, polyethylene glycol; Values are mean \pm standard deviation or $\mathrm{n}$.

Table 2 Adverse events associated with bowel preparation

\begin{tabular}{|c|c|c|c|}
\hline Variable & $\begin{array}{l}\text { Mirabilite } \\
(n=19)\end{array}$ & $\begin{array}{l}\text { PEG } \\
(n=21)\end{array}$ & $P$ value \\
\hline Nausea & 7 (36.84\%) & $16(76.19 \%)$ & 0.024 \\
\hline Vomiting & 0 & $7(33.33 \%)$ & 0.009 \\
\hline Bloating & 3 (15.79\%) & 0 & 0.475 \\
\hline Abdominal pain & $1(5.26 \%)$ & 0 & 0.098 \\
\hline Dizziness & 3 (15.79\%) & $1(4.76 \%)$ & 0.331 \\
\hline Headache & $1(5.26 \%)$ & 0 & 0.475 \\
\hline Chest distress & 0 & $2(9.52 \%)$ & 0.489 \\
\hline
\end{tabular}

PEG, polyethylene glycol; Values are n (\%).

Table 3 Abundant taxa in mirabilite preparation group

\begin{tabular}{|c|c|c|c|c|c|c|}
\hline \multirow[t]{2}{*}{ Taxa } & \multicolumn{2}{|c|}{ Lavage with Mirabilite } & \multicolumn{2}{|c|}{ Placebo (M1 group) } & \multicolumn{2}{|c|}{ Probiotics (M2 group) } \\
\hline & Baseline & day 1 & day 14 & day 28 & day 14 & day 28 \\
\hline \multicolumn{7}{|l|}{ Firmicutes/Clostridia } \\
\hline Anaerostipes_hadrus & $340.5(200.0)$ & $491.1 *(214.0)$ & $528.7 *(237.3)$ & 275.7(178.9) & $358.8(20.1 .2)$ & $511.1(365.0)$ \\
\hline Butyricicoccus & $307.6(239.3)$ & $555.0 *(734.5)$ & $543.9 *(341.6)$ & $248.9(127.9)$ & $244.3(79.7)$ & 236.2(98.2) \\
\hline Romboutsia ilealis & 194.3(138.4) & $330.1 *(219.1)$ & $398.0 *(225.5)$ & 201.2(132.0) & $290.1(265.3)$ & 184.2(101.4) \\
\hline Ruminococcus_torques_group & $257.7(170.6)$ & $413.4 *(204.6)$ & $467.6 *(260.6)$ & $305.1(142.3)$ & $140.7(80.0)$ & $273.2(264.3)$ \\
\hline \multicolumn{7}{|l|}{ Bacteroidota/Bacteroidia } \\
\hline
\end{tabular}


Solid blue color is indicative of significantly higher abundance compared to baseline, respectively. Values are mean (standard deviation). Statistical difference was determined by a Wilcoxon rank sum test. $\mathrm{p}<0.05$ is marked with an asterisk.

Table 4 Abundant taxa in PEG preparation group

Taxa Lavage with PEG Placebo (P1 group) Probiotics (P2 group)

\begin{tabular}{|c|c|c|c|c|c|c|}
\hline & & & & & & \\
\hline & Baseline & day 1 & day 14 & day 28 & day 14 & day 28 \\
\hline Firmicutes/Bacilli & & & & & & \\
\hline Holdemanella & $85.3(75.5)$ & $193.6 *(173.9)$ & $77.9(46.8)$ & $141.1(145.0)$ & $192.5^{\star}(148.5)$ & $277.9(486.7)$ \\
\hline Lactobacillus_reuteri & $0(0)$ & $54.2 *(113.3)$ & $41.2 *(61.3)$ & $0.18(0.60)$ & $0(0)$ & $0.09(0.30)$ \\
\hline Firmicutes/Clostridia & & & & & & \\
\hline Blautia & $834.8(301.6)$ & $1122.0 *(414.7)$ & $804.4(283.5)$ & $1051.0(558.5)$ & $800.3(276.1)$ & $732.7(744.6)$ \\
\hline Ruminococcus_torques_group & $224.6(80.2)$ & $380.6 *(225.4)$ & $285.1(187.2)$ & $237.5(167.7)$ & $280.6(136.5)$ & $128.9 *(97.4)$ \\
\hline UCG_002 & $282.5(134.8)$ & $160.0 *(87.9)$ & $170.5^{\star}(170.1)$ & $176.5^{*}(94.9)$ & $608.3 *(562.0)$ & $277.0(171.5)$ \\
\hline Bacteroidota/Bacteroidia & & & & & & \\
\hline Bacteroides_uniformis & $644.4(487.8)$ & $403.3 *(329.2)$ & $505.4(662.8)$ & $827.5(707.1)$ & $473.9(294.7)$ & $427.7(524.1)$ \\
\hline Bacteroides_caecigallinarum & $0(0)$ & $116.6 *(301.2)$ & $294.5^{\star}(461.1)$ & $0(0)$ & $0(0)$ & $0(0)$ \\
\hline Fusobacteriota/Fusobacteriia & & & & & & \\
\hline Fusobacterium_mortiferum & $73.62(183.7)$ & $752.2^{\star}(2361)$ & $1155.0(3524.0)$ & $596.7(1510.0)$ & $100.0(200.0)$ & $60.60(190.2)$ \\
\hline
\end{tabular}

Solid blue color and gray color are indicative of significantly higher abundance and lower abundance compared to baseline, respectively. Values are mean (standard deviation). Statistical difference was determined by a Wilcoxon rank sum test. $p<0.05$ is marked with an asterisk.

\section{Figures}

\section{$\checkmark \quad$ Sample collection}

Lavage
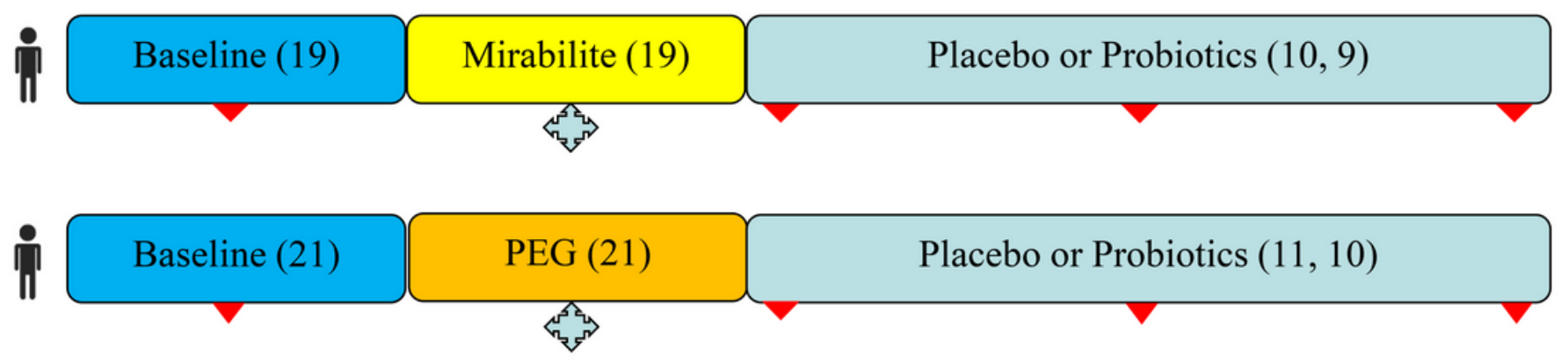
Day -1
Day 0
Day 1
Day 14
Day 28

Figure 1 
A PEG and Placebo

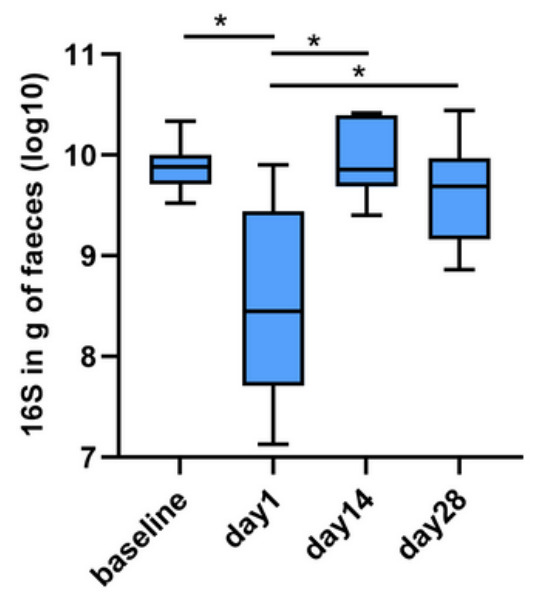

C Mirabilite and Placebo

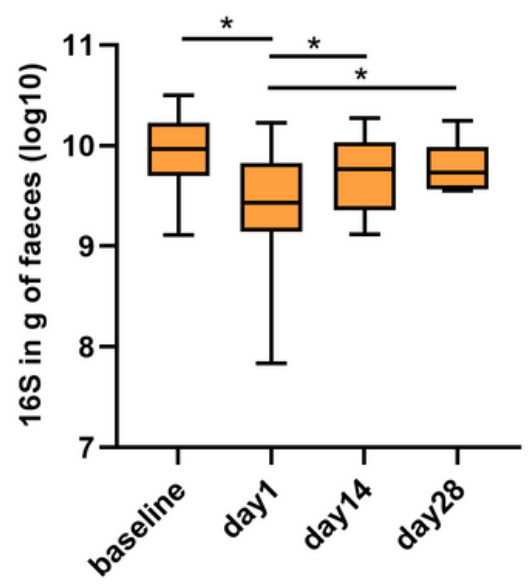

B

PEG and Probiotics

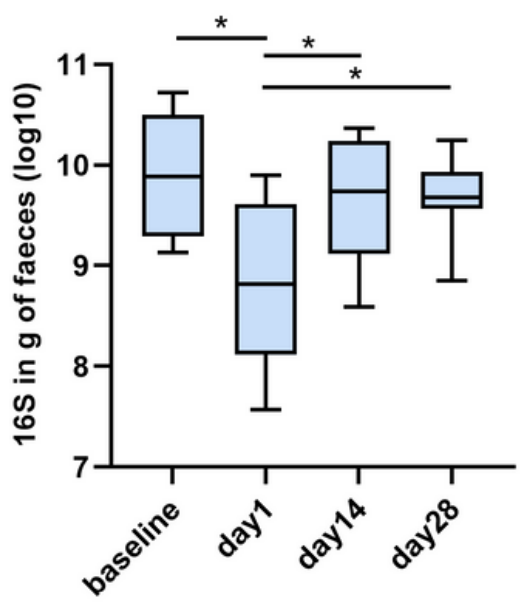

D Mirabilite and Probiotics

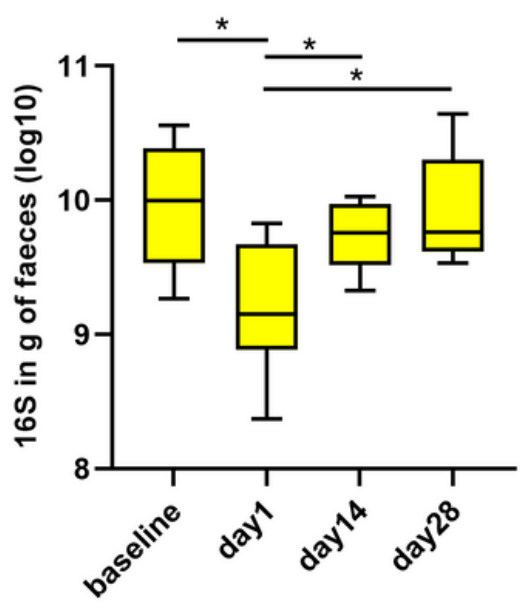

E

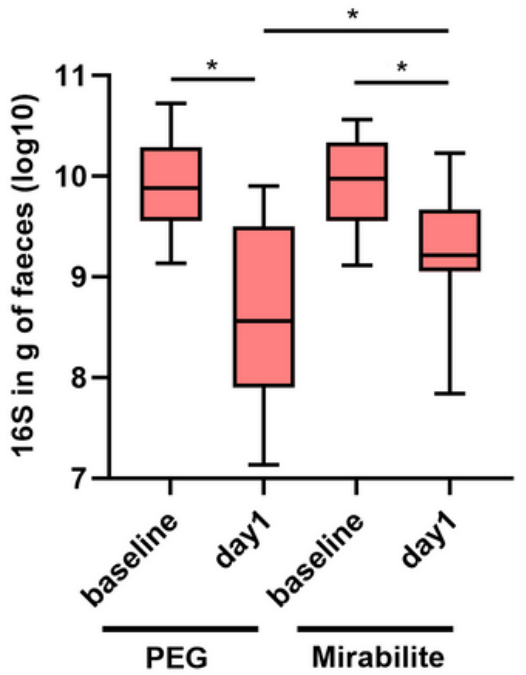

F

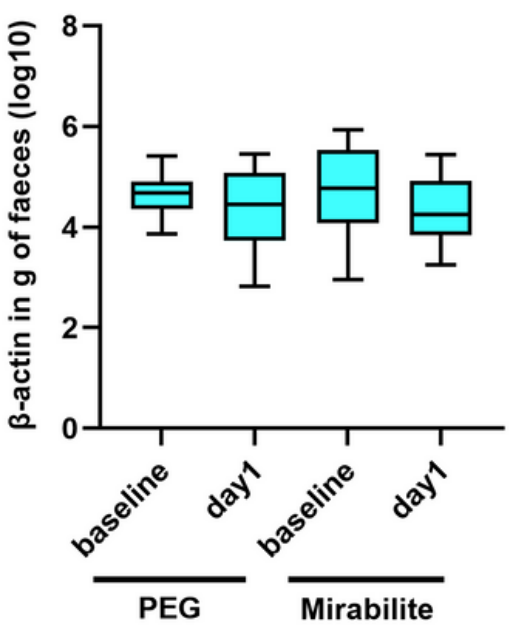

Figure 2

The effects of bowel cleansing on total bacterial load. (A-D) The total bacteria number at fecal samples between baseline and different followup time points in each experimental group. (E) Comparison of effects of mirabilite and PEG preparation on total bacteria load. (F) Quantitative analysis of human gene (beta-actin) at fecal samples between baseline and 1 day of follow-up in mirabilite and PEG preparation group. Total bacteria number and beta-actin were measured with quantitative PCR. Independent samples t-test was used to determine the differences between sample groups, and $p<0.05$ is indicated with an asterisk. 
A

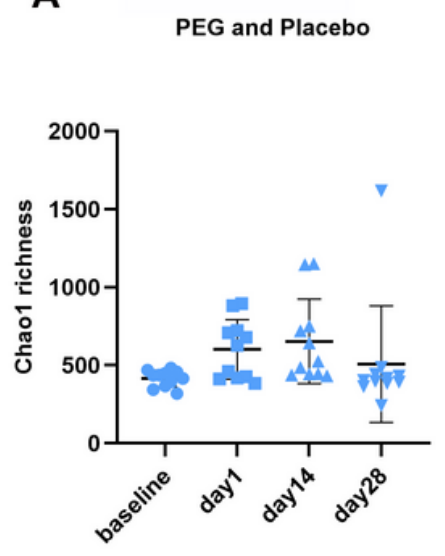

E

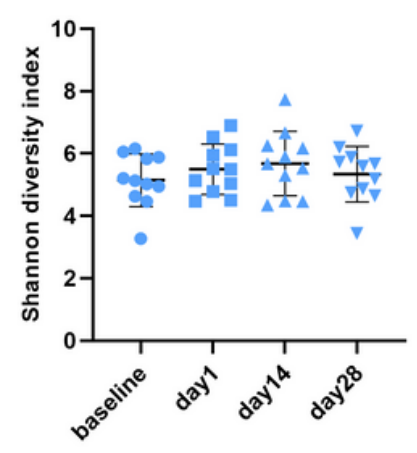

B

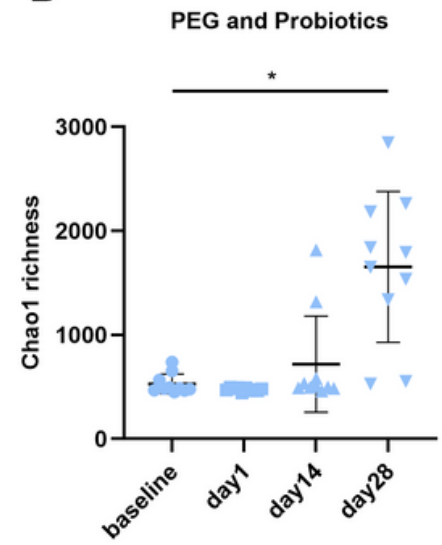

$\mathbf{F}$

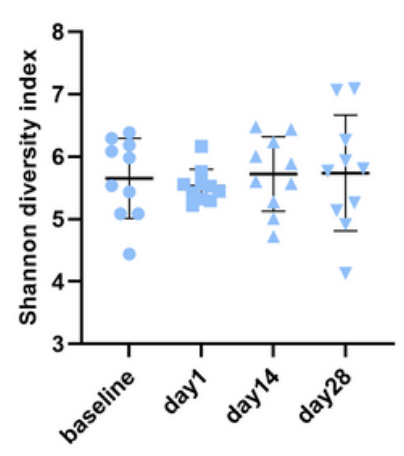

C

Mirabilite and Placebo

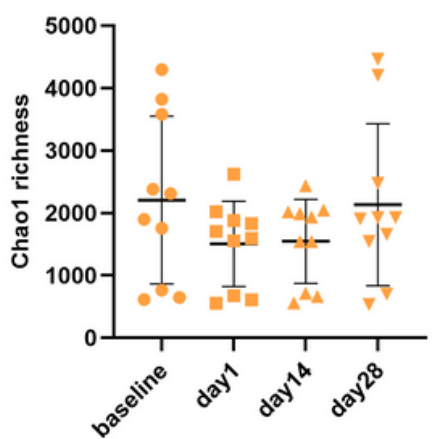

G

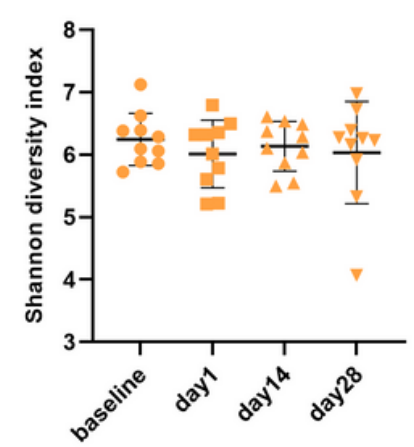

D Mirabilite and Probiotics

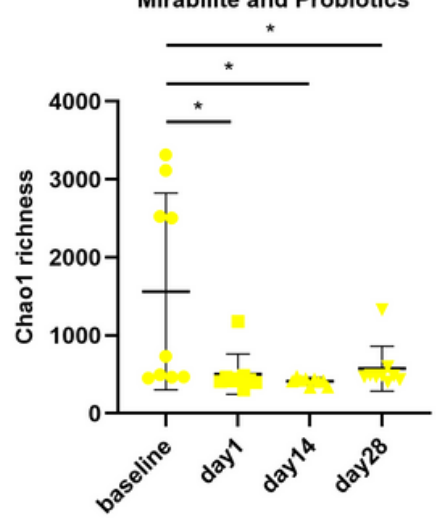

H

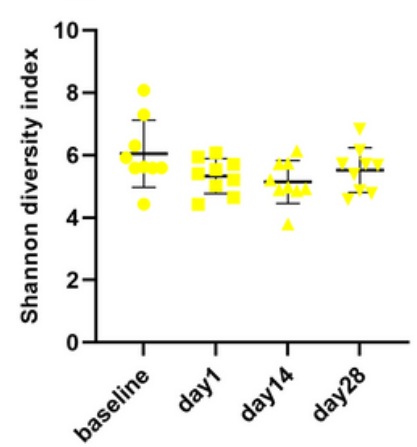

Figure 3

Alpha-diversity assessed by richness and diversity. (A-D) Chao1 richness in each experimental group. (E-H) Shannon diversity index in each experimental group. Each spot represents a single sample. A Wilcoxon rank sum test was used to determine the differences between sample groups, and $p<0.05$ is indicated with an asterisk. 
A

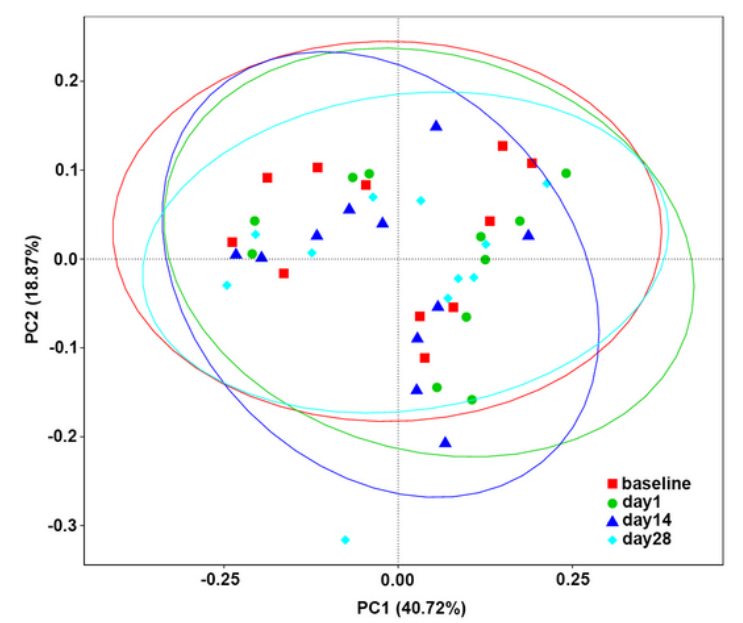

C

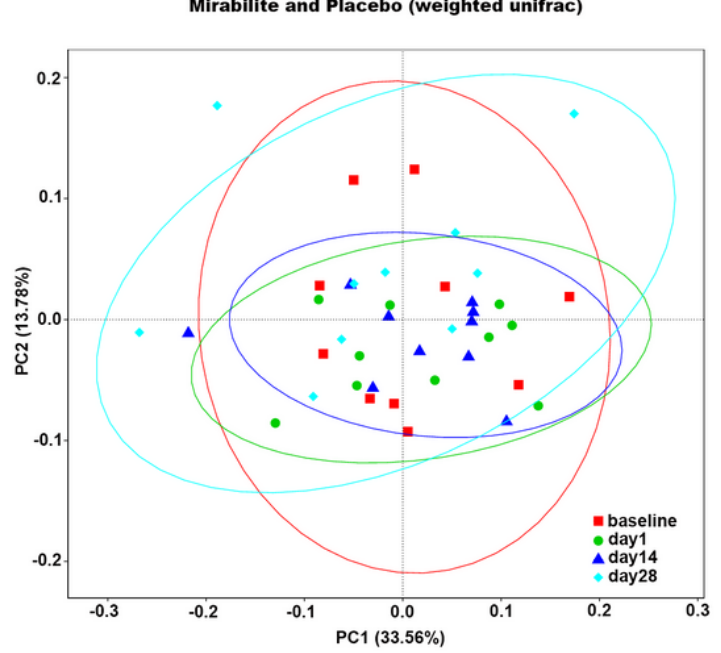

B

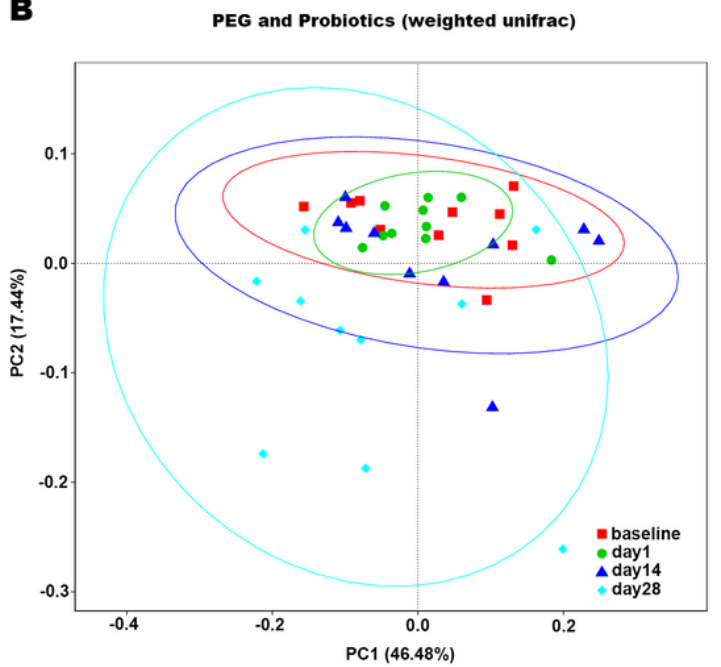

D

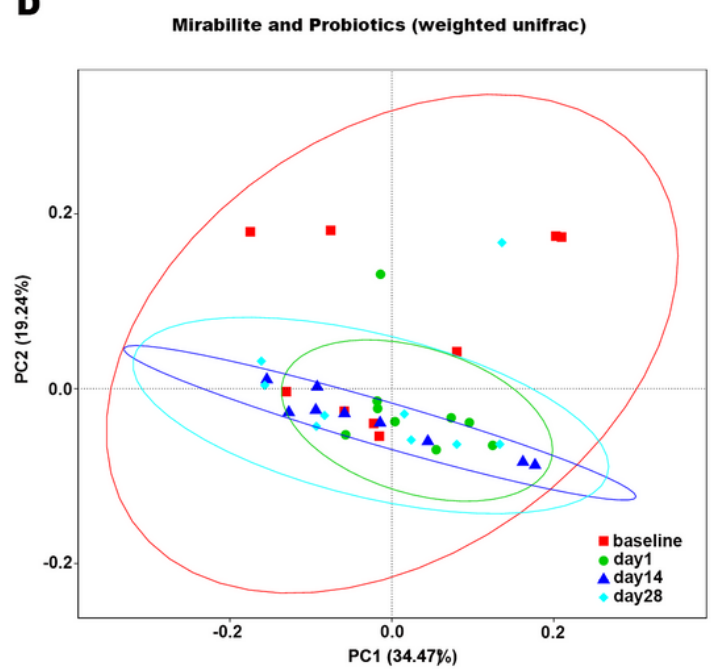

\section{Figure 4}

Principal coordinate analysis ( $\mathrm{PCOA}$ ) based on weighted unifrac distance matrices in each experimental group. Each spot represents a single sample. Differences in microbiota composition between sample groups were determined by adonis test. Ellipses represent an $80 \%$ confidence interval. Color/shape is indicative of sample group. 
$\mathbf{A}$

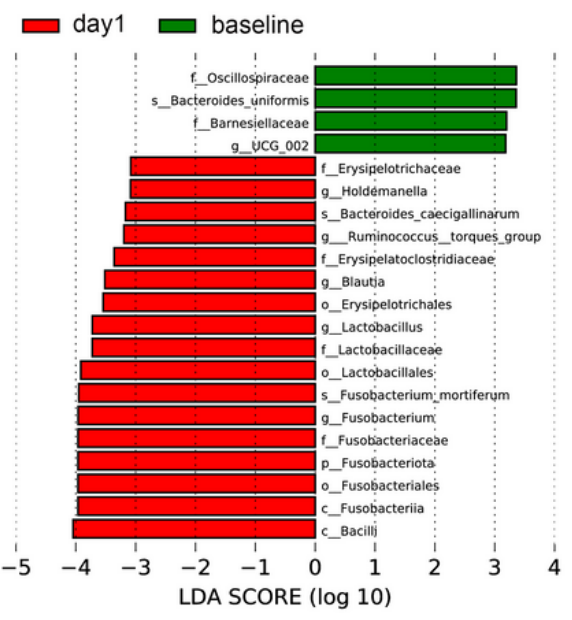

C

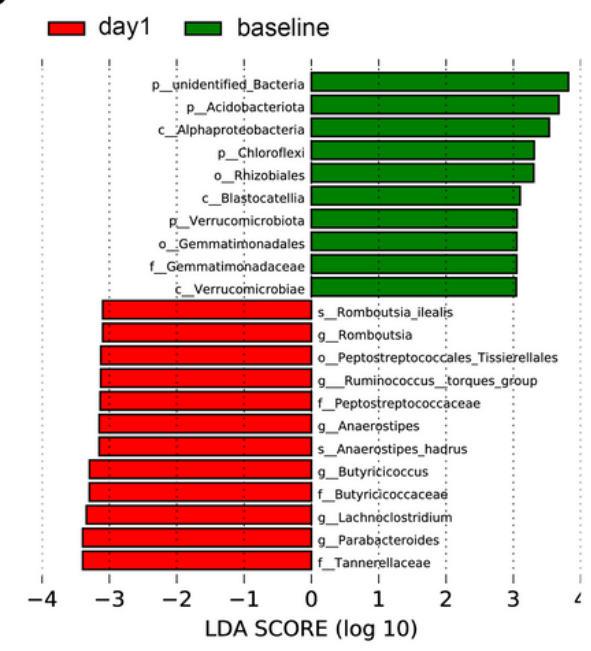

B

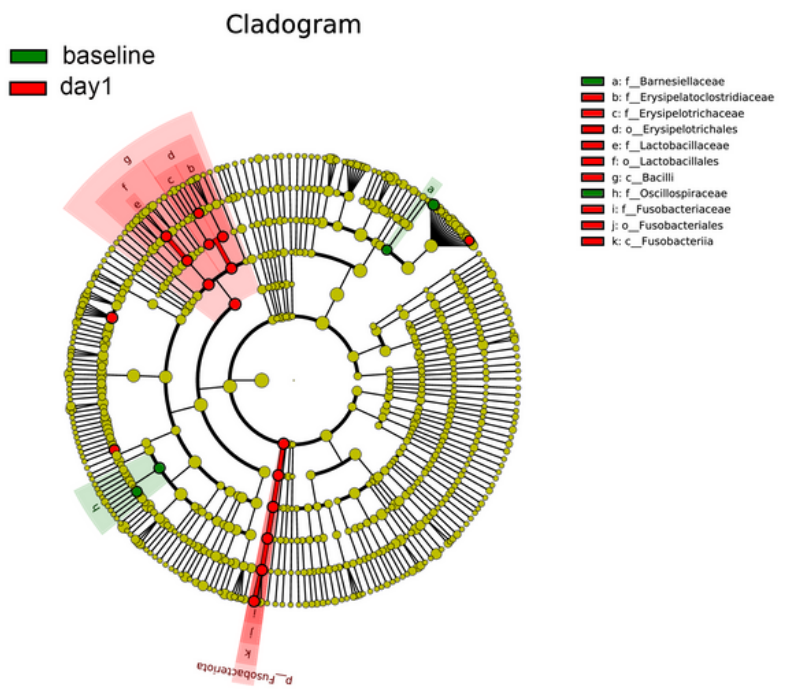

D

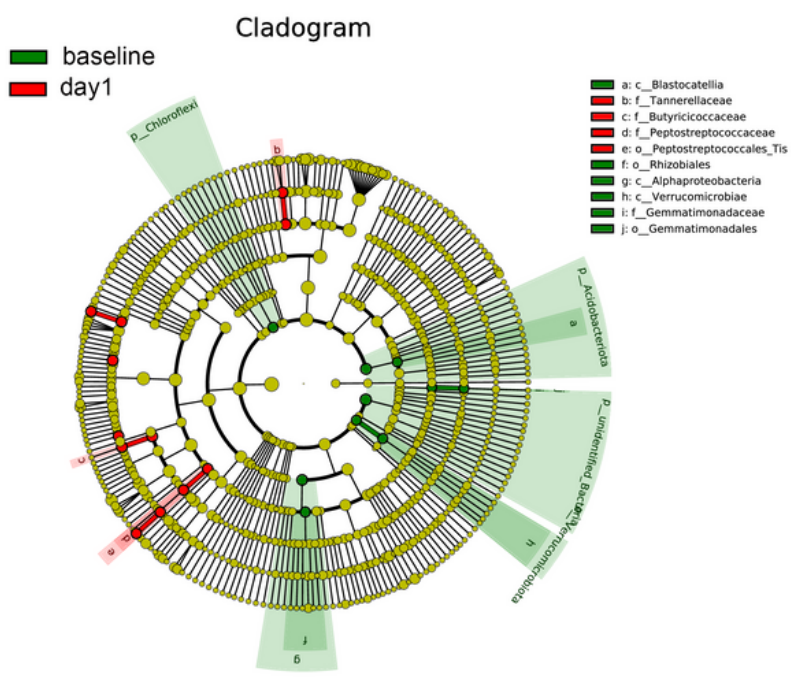

\section{Figure 5}

Linear discriminant analysis Effect Size (LEfSe) analysis of microbial abundance among different sample groups. Taxa with significantly difference at samples between baseline and 1day of follow-up in PEG preparation group (A), and in mirabilite preparation group (C) were displayed (LDA score>3, p<0.05). p, phylum; c, class; o, order; f, family; g, genus. Cladogram exhibited the phylogenetic distribution between baseline and 1day of follow samples in PEG preparation group (B), and in mirabilite preparation group (D). Red and blue nodes/shades indicate that taxa are significantly higher abundant in each group.

\section{Supplementary Files}

This is a list of supplementary files associated with this preprint. Click to download.

- FigureS1.tif

- Figures2.tif

- Figures3.tif

- Figures4.tif

- Tables1.docx

- Tables2.docx

- Tables3.docx

- Tables4.docx 